\title{
BMJ Open Improvement of transitional care from hospital to home for older patients, the TIGER study: protocol of a randomised controlled trial
}

\author{
Martina Rimmele (D) , ${ }^{1}$ Jenny Wirth, ${ }^{1}$ Sabine Britting, ${ }^{1}$ Thomas Gehr, ${ }^{1,2}$ \\ Margit Hermann, ${ }^{3}$ Dirk van den Heuvel, ${ }^{4}$ Andreas Kestler, ${ }^{2}$ Thomas Koch, ${ }^{5}$ \\ Oliver Schoeffski, ${ }^{6}$ Dorothee Volkert, ${ }^{1}$ Klaus Wingenfeld, ${ }^{7}$ Susanne Wurm, ${ }^{8}$ \\ Ellen Freiberger (D) , ${ }^{1}$ Cornel Sieber, ${ }^{1,9}$ TIGER consortium
}

To cite: Rimmele M, Wirth J, Britting S, et al. Improvement of transitional care from hospital to home for older patients, the TIGER study: protocol of a randomised controlled trial. BMJ Open 2021;11:e037999. doi:10.1136/ bmjopen-2020-037999

- Prepublication history and additional material for this paper is available online. To view these files, please visit the journal online (http://dx.doi.org/10. 1136/bmjopen-2020-037999).

Received 24 February 2020 Revised 25 November 2020 Accepted 02 December 2020

A Check for updates

(c) Author(s) (or their employer(s)) 2021. Re-use permitted under CC BY-NC. No commercial re-use. See rights and permissions. Published by BMJ.

For numbered affiliations see end of article.

Correspondence to Dr Martina Rimmele; martina.rimmele@fau.de; merimmele@yahoo.com

\section{ABSTRACT}

Introduction In Germany, an efficient and feasible transition from hospital to home for older patients, ensuring continuous care across healthcare settings, has not yet been applied and evaluated. Based on the transitional care model (TCM), this study aims to reduce preventable readmissions of patients $\geq 75$ years of age with a transitional care intervention performed by geriatric-experienced care professionals. The study investigates whether the intervention ensures continuous care during transition and stabilises the care situation of patients at home.

Methods and analyses Randomised controlled clinical trial, recruiting between 25 April 2018 and 31 December 2019 in one German hospital in the city of Regensburg. The intervention group is supported by care professionals in the transition process from hospital to home for up to 12 months. Based on TCM, the intervention includes an individual care plan according to a patient's symptoms, risks, needs and values. The plan is advanced in the domestic situation via personal visits and telephone contacts. All necessary care actions regarding, for example, mobility, residence adjustments, or nutrition, are initiated to be executed by ambulant care services, and are monitored, evaluated and adapted if necessary. In supervising the care plan, the care professionals do not administer active care services themselves but coordinate them. Patients and their caregivers are actively engaged in the care planning and execution. In contrast, the control group receives only usual discharge planning in the hospital and usual ambulatory care.

The primary outcome is the all-cause readmission rate assessed using health insurance data within a follow-up of up to 12 months after hospital discharge. Secondary outcomes include care quality, mobility, nutritional and wound situation, and health-related quality of life. They are assessed at baseline, after 1 month, 3 months, 6 months, and at the end of study visit. Additionally, the economic efficiency of the intervention will be evaluated.

Ethics and dissemination Ethics approval for the trial was obtained from the Ethics Committee of the FriedrichAlexander-Universität Erlangen-Nürnberg. Results will be published in peer-reviewed, open-access scientific
Strengths and limitations of this study

- The findings of the trial will help to define so far missing recommendations for the implementation of efficient transsectoral care for older patients in the German healthcare system.

- Transitional care professionals in the study offer a potential long-term benefit for home-dwelling patients, supporting them for up to 12 months.

- The intervention is highly individualised to each participant's needs and situation, but overall addressing and evaluating the importance of a broad spectrum of aspects, such as mobility, nutrition and care quality.

- In case of readmission of a participant, the care professional promotes continuity of care and information transfer from the ambulant care setting back into the hospital, but due to the setting, this conclusion can only be drawn for the hospital of the study.

- Patients with cognitive deficits might profit from our intervention; however, only individuals with a MiniMental State Examination Score of at least 22 points are included to ensure that participants are able to benefit from the self-management approach of the TCM.

journals and disseminated at national and international research conferences and through public presentations in the geriatric and healthcare community.

Trial registration ClinicalTrials.gov identifier: NCT03513159.

\section{INTRODUCTION}

Considerable medical progress and improved living conditions in the last decades have enabled an increasing life expectancy, leading to a marked ageing of the population in Europe. ${ }^{1}$ Expanded life expectancy is, however, often accompanied by multimorbidity, poorer quality of life, loss of function, polypharmacy and inconsistencies in care, ${ }^{23}$ 
resulting in increased hospital admissions. ${ }^{4}$ Moreover, in Germany, approximately $30 \%$ of hospital patients who are $\geq 70$ years old, show a hospital-associated impairment in activities of daily living (ADL) and health-related quality of life at hospital discharge. ${ }^{5}$ Mobility and nutritional status are especially prone to deteriorate during hospital stays in older patients ${ }^{6}$ and can lead to rehospitalisations. In addition, patients with chronic wounds (eg, diabetic feet) run the risk of poor wound healing and wound care during transition from hospital to home. Moreover, the Diagnosis-Related Groups hospital payment system, promoting a shortening of hospitalisation days to reduce costs, enhances the risk for early unplanned rehospitalisations. ${ }^{7}$ As reported in 2015, in $20 \%$ of Medicare beneficiaries in the USA, readmissions occur within 30 days of discharge and in $34 \%$ within 90 days, leading not only to additional loss of independence of patients, but also to additional costs for the healthcare system. ${ }^{8}$ Thus, the USA instituted the Hospital Readmissions Reduction Programme holding hospitals accountable for unnecessary rehospitalisations. ${ }^{10}$

In Germany, as opposed to the mostly private sector healthcare of the USA, healthcare builds on a socialised compulsory insurance system. Generally, citizens either join a public or private health insurance fund. Public healthcare organisation and financing are under joint self-government of physicians, health insurances, hospitals and insurees, and are performed by private and public providers. This led to the development of fragmented, rigid, consolidated structures with separate in-hospital and ambulatory care programmes and reimbursement options, causing particular problems to bridge the gap between hospital and ambulatory care efficiently and from one source. To address deficiencies in the transition process from inpatient to homesetting, the German government amended a law in 2012 to improve healthcare structures and to strengthen the patients' rights for discharge planning ( 39 SGB V). However, hospital discharge planning is not sufficient to guarantee the patients' readaptation and well-being at home after hospital discharge. ${ }^{11}{ }^{12}$ It has been reported that the implementation of actions initiated by discharge teams could not be verified at home, arranged adaptations not audited and interruptions in the continuity of care not identified. ${ }^{1314}$ Family physicians point to an unmet need for medically competent contact persons inside hospitals to efficiently communicate medical issues regarding patients transferring from hospital to home. ${ }^{15}$ Relevant information might be lost, provoking health deterioration and conceivably preventable readmissions. ${ }^{1516}$

A distinct need for action was declared in Germany for older people with multimorbidity, notably for people 80 years of age and above. They experience increased vulnerability through complications, secondary diseases, chronic conditions, heightened risks of autonomy loss as well as a loss of self-help capacity facing increased hospitalisations. ${ }^{17}$
Applying transitional care programmes aiming at patients with high risk for poor outcomes and readmissions, such as older people with multimorbidities and complex chronic diseases can reduce preventable readmissions by up to $75 \% .^{18-24}$

The most rigorously tested model that has consistently demonstrated effectiveness to promote safe, timely and appropriate transfer between settings comprising education interventions, pre-discharge evaluation and patientcentred care at home, is the transitional care model (TCM). ${ }^{21} 22$ 25-27

This is the first large randomised controlled trial in Germany that aims to evaluate an efficient and feasible care transition from hospital to home for older geriatric patients $\geq 75$ years of age, based on $\mathrm{TCM},{ }^{22}$ and not disease oriented. It integrates discharge planning in the hospital with ambulant healthcare providers such as family physicians and ambulant care services to sustain patient care continuity across settings. The study investigates geriatric patients in a semi-rural setting in southern Germany, so cultural aspects could influence the transitional process from hospital to home.

Since academically educated advanced nurse practitioners are only starting to be trained in Germany, the intervention is performed by geriatric-experienced care professionals embedded in a team combining complementary expertises. As the care professionals find and guide the path for patients for better care, they are called 'pathfinders'.

\section{Objectives}

The main objective is to improve geriatric care at the transition from hospital to home, reflected by a reduction of the all-cause readmission rate within a follow-up of up to 12 months after hospital discharge (primary outcome) in a randomised controlled trial. We hypothesise that the TCM-based intervention performed by geriatricexperienced care professionals will achieve a readmission reduction by improving the care situation of the patients at home and according to their housing and caregiving situation, contributing to a stable or improved state of their mobility, functionality, nutrition, wound healing, independence and health-related quality of life while reducing costs.

The effects of the intervention will be analysed and its efficacy and feasibility evaluated to be able to make recommendations on which parts or activities of this TCM-concept intervention might be implemented in the German healthcare system.

\section{METHODS}

\section{Trial design and study setting}

The Transsectoral Intervention Programme for Improvement of Geriatric Care in Regensburg (TIGER) study is a randomised controlled clinical trial with an intervention and a control group (see figure 1). It takes place at the St John of God Hospital, Regensburg ('Barmherzige Brüder 
Screening and recruiting leading to Enrollment $(n \geq 280)$

Inclusion criteria: being $\geq 75$, going back home, AOK-health insurance, MMSE $\geq 22$, residence within $\leq \mathbf{5 0} \mathbf{~ k m}$ of hospital

Exclusion criteria: palliative, planned readmission within 4 weeks

Patients will have a different length of stay in the study, depending on the date of recruitment:

Recruitment April 2018 to end of June 2019: 12 months

Recruitment July 2019 to end of September 2019: 9 months

Recruitment October 2019 to end of December 2019: 6 months

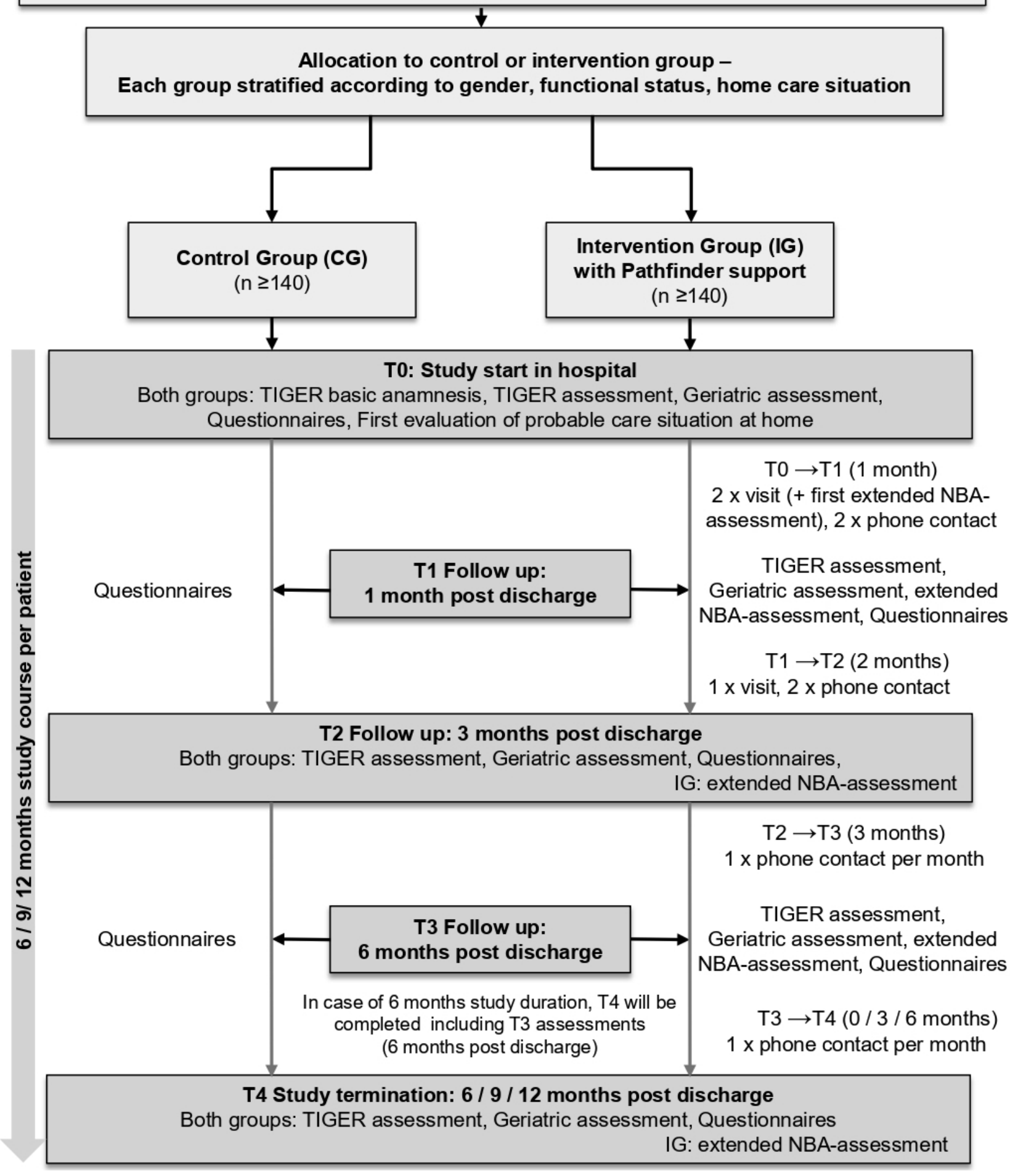

Figure 1 Study design. MMSE, Mini-Mental State Examination; NBA, Neues Begutachtungs Assessment, an assessment to determine eligibility for benefits from the long-term care insurance in Germany ${ }^{31}$; TIGER, Transsectoral Intervention Programme for Improvement of Geriatric Care in Regensburg 
Pre-phase

Study-phase: Recruiting and intervention

Evaluation/ Dissemination

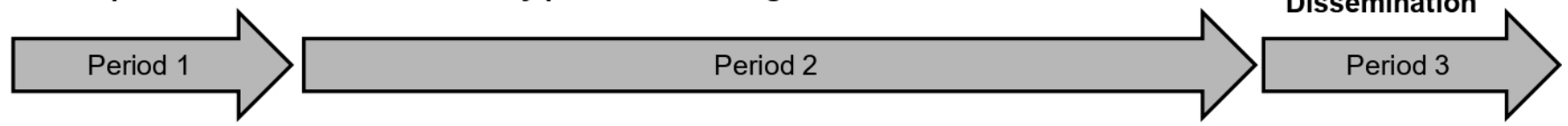

- Development of study protocol of operations

- Development of recruitment and documentation material

- Development of eCRF

- Structural processes preparations in hospital

- Staff recruitment

- Training course for TIGER staff
- First patient in on $25^{\text {th }}$ April 2018

- Recruitment and recruiting optimizations

- Pathfinder activities, precise documentation of each activity and needed time for it

- Data collection for scientific accompanying research

- Monitoring of quality of intervention and data collection

- Process improvements, protocol of operations amendments

- 6 / 9 / 12 months study course per patient, depending on recruitment date

- T0-T4 visits in intervention and control group with assessment of secondary outcomes
- Statistical and qualitative evaluation of primary (readmission rate) and secondary outcomes

- Health economic evaluation

- Initiation of dissemination process

- Publication of results

Figure 2 Timeline of overall TIGER project. eCRF, electronic case report form; TIGER, Transsectoral Intervention Programme for Improvement of Geriatric Care in Regensburg.

Regensburg', BBR), Germany, in the city of Regensburg and surroundings. Since the effectiveness and feasibility of the application of a transitional care intervention itself will be evaluated in the study, the intervention is focusing on one hospital setting. The timeline of the overall TIGER project is depicted in figure 2 .

\section{Participant timeline}

The study duration per participant is at least 6 months, and in the case of early recruitment (before end of June 2019) 12 months (see figure 1). Since recruitment was lagging, as also reported in other clinical trials engaging persons over 65 years, ${ }^{28}$ we prolonged the recruitment phase from initially planned 12 months to 20 months to reach the calculated sample size. The follow-up visit plan had to be adapted, since the end of the overall study intervention phase could not be prolonged proportionately due to project funding reasons. The intervention period is planned to end on 30 June 2020. In figure 1, the timeline for each participant according to his recruitment date is illustrated with all visit times T0-T4. The schedule of intervention and control group assessments is presented in table 1 .

\section{Eligibility criteria}

Male and female patients from all wards of BBR, aged 75 years or older, and being insured by the statutory health insurance AOK (Allgemeine Ortskrankenkasse) Bavaria are eligible for this study. Patients need to reside within a radius of $50 \mathrm{~km}$ distance to the hospital, return to their home environment after discharge and reach a MiniMental State Examination (MMSE) ${ }^{29}$ result of at least 22 points. Although patients with less than 22 points in the MMSE might also benefit from the intervention, we chose this threshold to ensure that participants will be able to benefit from the self-management approach of the TCM, and to fill out questionnaires themselves. Exclusion criteria are palliative care situation (defined by the statement 'therapeutic goal: palliative' (instead of curative) in the medical report) and planned readmission to the hospital within the next 4 weeks.

\section{Recruitment process}

A TIGER-specific IT tool supported screening for potential participants according to the eligibility criteria age, health insurance and residence within a $50 \mathrm{~km}$ radius electronically in all wards via the patient management system of BBR. All patients admitted between April 2018 and December 2019 were scanned by this tool. Potential participants identified by this tool were visited in person by the TIGER staff who assessed all other eligibility criteria and informed about the project. Patients in BBR fulfilling all eligibility criteria and present caregivers were then provided with the participant information brochure and informed consent form (ICF) (see online supplemental file 1). Patients were given at least 1 day to read the provided information and informed consent form and receive further information on the project. After signing and dating the informed consent form, the $\mathrm{MMSE}^{29}$ was performed as a last inclusion criterion for recruitment.

\section{Randomisation}

Stratified block randomisation was performed with the following three strata': (1) gender (male/female), (2) mobility (can walk at least four stair steps: yes/no) ${ }^{30}$ and (3) living condition (lives alone: yes/no). These strata were chosen because of their potential to influence the overall need for care and study outcomes.

The randomisation blocks varied between 2, 4 and 6 to guarantee a minimum of predictability for the randomisation. When receiving a recruitment number for a newly recruited participant in the electronic data acquisition and electronic case report form (eCRF) System (secuTrial $^{\circledR}$ ), the stratification questions had to be answered, the inclusion criteria affirmed and the exclusion criteria denied in the eCRF file. Then, the randomisation into intervention or control group was performed automatically by the eCRF system.

\section{Study staff and training}

Academically educated advanced nurse practitioners are not available yet in Germany. The study is thus performed by geriatric-experienced care professionals 
Table 1 Visit plan and standardised assessments of participants

\begin{tabular}{|c|c|c|c|c|c|c|c|c|c|c|}
\hline \multirow[b]{2}{*}{$\begin{array}{l}\text { All participants (both } \\
\text { groups) standardised } \\
\text { assessments }\end{array}$} & \multicolumn{2}{|c|}{$\begin{array}{l}\text { T0 (at day of } \\
\text { discharge) }\end{array}$} & \multicolumn{2}{|c|}{ T1 (after 1 month) } & \multicolumn{2}{|c|}{ T2 (after 3 months) } & \multicolumn{2}{|c|}{ T3 (after 6 months ${ }^{\star}$ ) } & \multicolumn{2}{|c|}{$\begin{array}{l}\text { T4 (end of study } \\
\text { visit*) }\end{array}$} \\
\hline & CG & IG & $\begin{array}{l}\text { CG (no } \\
\text { visit) }\end{array}$ & IG & CG & IG & $\begin{array}{l}\text { CG (no } \\
\text { visit) }\end{array}$ & $I G$ & CG & $I G$ \\
\hline $\begin{array}{l}\text { Initial assessment of } \\
\text { diagnoses and care } \\
\text { situation as usual in } \\
\text { hospital }\end{array}$ & $x$ & $x$ & - & - & - & - & - & - & - & - \\
\hline \multicolumn{11}{|l|}{ TIGER basic anamnesis: } \\
\hline $\begin{array}{l}\text { Hospital admission } \\
\text { situation, and social, } \\
\text { housing, care and } \\
\text { medication situation }\end{array}$ & $x$ & $x$ & - & - & - & - & - & - & $x$ & $x$ \\
\hline $\begin{array}{l}\text { Initial assessment of } \\
\text { domestic care situation } \\
\text { at first visit at home (in IG } \\
\text { between T0 and T1, in CG } \\
\text { at T2), modified according } \\
\text { to NBA† of }{ }^{31}\end{array}$ & - & - & - & $x$ & $x$ & - & - & - & - & - \\
\hline
\end{tabular}

\section{TIGER assessments:}

\begin{tabular}{|c|c|c|c|c|c|c|c|c|c|c|}
\hline $\begin{array}{l}\text { Mobility assessment } \\
\text { according to } \mathrm{NBA}^{31}\end{array}$ & $x$ & $x$ & - & $x$ & $x$ & $x$ & - & $x$ & $x$ & $x$ \\
\hline $\begin{array}{l}\text { Physical function: short } \\
\text { physical performance } \\
\text { battery } 39\end{array}$ & $x$ & $x$ & - & $x$ & $x$ & $x$ & - & $x$ & $x$ & $x$ \\
\hline $\begin{array}{l}\text { Nutrition status: mini } \\
\text { nutritional assessment }{ }^{33} 34\end{array}$ & $x$ & $x$ & - & $x$ & $x$ & $x$ & - & $x$ & $x$ & $x$ \\
\hline $\begin{array}{l}\text { Anthropometrics (weight } \\
\text { and size) }\end{array}$ & $x$ & $x$ & - & $x$ & $x$ & $x$ & - & $x$ & $x$ & $x$ \\
\hline $\begin{array}{l}\text { Cognitive assessment: trail } \\
\text { making test } A \text { and } B^{40}\end{array}$ & $x$ & $x$ & - & - & $x$ & $x$ & - & - & $x$ & $x$ \\
\hline $\begin{array}{l}\text { Handgrip strength via } \\
\text { dynamometer (Jamar } \\
\text { digital hand dynamometer, } \\
\text { model number 081406453) }\end{array}$ & $x$ & $x$ & - & $x$ & $x$ & $x$ & - & $x$ & $x$ & $x$ \\
\hline $\begin{array}{l}\text { Pedometer assessment: } \\
\text { activPAL3 micro (PAL } \\
\text { Technologies), worn for } 7 \\
\text { consecutive days }\end{array}$ & $x$ & $x$ & - & - & $x$ & $x$ & - & - & $x$ & $x$ \\
\hline
\end{tabular}

\section{Geriatric assessment:}

\begin{tabular}{|c|c|c|c|c|c|c|c|c|c|c|}
\hline MMSE $^{29}$ & $x$ & $x$ & - & $x$ & $x$ & $x$ & - & $x$ & $x$ & $x$ \\
\hline Geriatric Depression & $x$ & $x$ & - & $x$ & $x$ & $x$ & - & $x$ & $x$ & $x$ \\
\hline
\end{tabular}

$$
\text { Scale } 32
$$

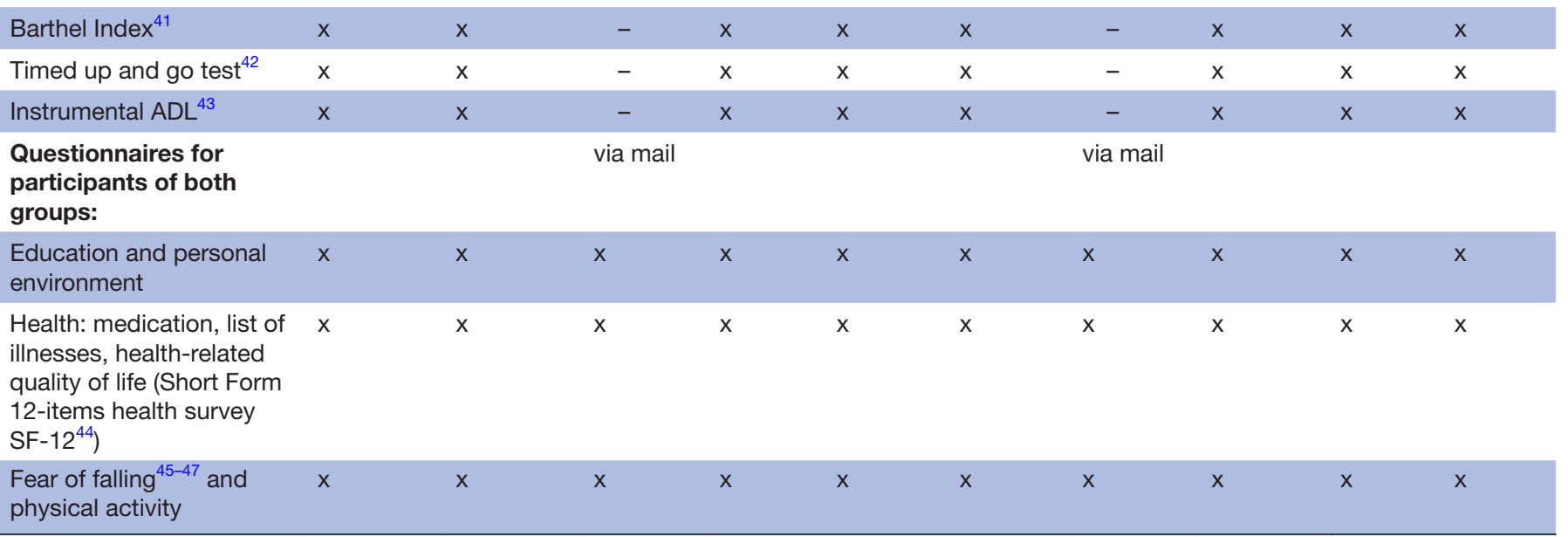




\begin{tabular}{|c|c|c|c|c|c|c|c|c|c|c|}
\hline \multirow[b]{2}{*}{$\begin{array}{l}\text { Personal psychosocial } \\
\text { resources regarding health } \\
\text { (ie, coping with disease, }^{48} \\
\text { self-perception of ageing } \\
\text { and self-efficacy }{ }^{50} \text { ) }\end{array}$} & \multicolumn{2}{|c|}{$\begin{array}{l}\text { T0 (at day of } \\
\text { discharge) }\end{array}$} & \multicolumn{2}{|c|}{ T1 (after 1 month) } & \multicolumn{2}{|c|}{ T2 (after 3 months) } & \multicolumn{2}{|c|}{ T3 (after 6 months*) } & \multicolumn{2}{|c|}{$\begin{array}{l}\text { T4 (end of study } \\
\text { visit*) }\end{array}$} \\
\hline & $x$ & $x$ & $x$ & $x$ & $x$ & $x$ & $x$ & $x$ & $\mathrm{x}$ & $x$ \\
\hline $\begin{array}{l}\text { Weight, diet, appetite, } \\
\text { food enjoyment }^{52} \text { and } \\
\text { support at meals }\end{array}$ & $x$ & $x$ & $x$ & $x$ & $x$ & $\mathrm{x}$ & $x$ & $x$ & $x$ & $x$ \\
\hline $\begin{array}{l}\text { Wound-related quality of } \\
\text { life }^{53}\end{array}$ & $x$ & $x$ & & & $x$ & $x$ & & & $x$ & $x$ \\
\hline $\begin{array}{l}\text { Perceived stress }{ }^{54} \text { and } \\
\text { caregiver burden }^{556}\end{array}$ & $x$ & $\mathrm{x}$ & $x$ & $\mathrm{x}$ & $\mathrm{x}$ & $\mathrm{x}$ & $\mathrm{x}$ & $\mathrm{x}$ & $\mathrm{x}$ & $x$ \\
\hline
\end{tabular}

*For participants with study duration of 9 months, the T4=end of study visit takes place 9 months after hospital discharge. For participants with study duration of 6 months, the T3 measurements taking place after 6 months is replaced by the T4=end of study visit measurements, but including all T3 assessments.

†The NBA is an assessment to determine eligibility for benefits from the long-term care insurance in Germany. ${ }^{31}$ ADL, activities of daily living; CG, control group; IG, intervention group; MMSE, Mini-Mental State Examination; NBA, Neues Begutachtungs Assessment; TIGER, Transsectoral Intervention Programme for Improvement of Geriatric Care in Regensburg.

called 'pathfinders', consisting of a registered nurse, a case manager, a head nurse and an occupational therapist, to combine and exchange if needed multiple expertises when addressing the broad spectrum of care need aspects, such as care quality, mobility and nutrition. Each pathfinder of the TIGER project is exclusively responsible for his TIGER participants. They are supported by the study physician. The staff is divided into pathfinders supporting the intervention group participants, and study nurses assessing the control group participants (see also table 1). To prevent contamination between the intervention and the control group in the hospital, all patients receive usual care as far as the non-TIGER hospital staff is concerned. Additional actions in the intervention group are initiated by the pathfinders.

The staff was trained in a 4-week TCM programme, including sessions on therapeutic and care requirements regarding physical performance, mobility and functionality, nutrition, wounds, the discharge planning of BBR, current legislation aspects of social law, health insurance and welfare opportunities by respective experts. The intervention programme with the necessary assessments, as well as the documentation, were taught and a detailed protocol of operations provided. During the study, weekly case conferences take place with all members. Pathfinders can seek and will obtain further training in specific diseases or care management topics throughout the study.

The study begins inside BBR and continues after discharge in the participants' homes for up to 12 months.

\section{Intervention and intervention group}

For the intervention group (IG), the pathfinders' activities are based on the TCM. ${ }^{22}$ The authors described 9 distinct but interdependent components in their programme, which may be combined both before and after discharge to achieve the best results for the participants. The TIGER intervention is based on all 9 components of the TCM (see table 2). For the German hospital and home setting of this study, however, the modules needed some adaptation due to German healthcare settings, work law and local requirements, as described in table 2.

\section{Intervention}

IG participants and their caregivers are accompanied by one pathfinder each in the process of hospital discharge, during the transition from hospital to home and for a minimum of 6 months up to 12 months after discharge (see figure 1). An individual care plan is developed by the designated pathfinder for each of the IG participants according to their symptoms, risks, needs and values (eg, physiotherapy, drug application and nutritional counselling) and in close collaboration with the care team (see table 2 'Collaborating', including the family physician). All care activities for the IG participants are initiated by the pathfinder within the care team. The pathfinder coordinates, monitors, evaluates, adapts, if necessary, and documents the execution of the activities and the participants' adherence. In developing the care plan, the pathfinders do not provide active care services themselves, but coordinate their execution by contacting ambulant services for the required service activities. For the project, it was essential to ensure that the pathfinders would not compromise the operational tasks of the usual ambulant services to be able to build a trusting relationship with these services. Participants and their caregivers are actively engaged in the care planning process. Progressively during the course of the intervention, self-management is promoted.

The family physicians of the IG participants are invited to actively take part in the study by the TIGER consortium partner Regensburger Aerztenetz, a network of family physicians in Regensburg, but this is no inclusion 
Table 2 Adaptation of TCM modules to the TIGER study intervention group in German healthcare setting

\section{Intervention module/component as defined in TCM according to Hirschman et $a l^{22}$}

(1) Screening of patients: screening for adults transitioning from hospital to home who are at high risk for poor outcomes

(2) Staffing for care planning and management: master's prepared APRN assume primary responsibility for care management of patients throughout episodes of acute illness

\section{(3) Maintaining relationships:} establishment and maintenance of a respectful, trusting relationship with the patient and family caregivers, including home visits, telephone calls, availability of the APRN in charge of the intervention 7 days a week

\section{Component adapted to setting in the TIGER study}

- For the randomised controlled TIGER study, a-specific IT tool supported screening for potential participants electronically via the patient management system of BBR according to the eligibility criteria age, health insurance and residence within a $50 \mathrm{~km}$ radius. All further eligibility assessment and recruitment was performed in person by the TIGER staff. Directly after recruitment, the participants were randomised to either intervention or control group via the electronic data acquisition and eCRF system.

- Four full-time care professionals with at least 5 years of care experience with geriatric patients, each of them with additional complementary skills for the team (registered nurses, occupational therapist, case manager and head nurse) and specifically trained for the intervention of this study are employed by BBR.

- Each IG patient is supported by one designated pathfinder during the intervention period. If complementary skills advice is needed, the pathfinder will find this within his pathfinders team or within the collaborating care team of his patient.

- Establishment of respectful, trusting relationships starts in the hospital (already prior to TO in the recruitment process) and is deepened throughout the intervention in home visits and telephone calls. A trusting relationship enables the identification of problems, needs, anxieties, as well as risks and symptoms.

- According to German working hour acts, the pathfinders will be available from Monday to Friday, not 7 days a week. The participants and their caregivers receive a telephone number of the pathfinder office, so that they can call the pathfinders with any occurring questions or problems. On weekends, when the office is closed, participants and their caregivers are instructed in detail how to leave a message on the pathfinder's answering machine and how to call the hospital's emergency department if immediate assistance is needed. On early Monday mornings, the pathfinders contact every person that has left a message on the answering machine to trace back everything that occurred over the weekend.

\section{(4) Engaging patients and caregivers} in care management planning and implementation:

engaging of patients and their caregivers in design and implementation of the plan of care aligned with their preferences, values and goals, in collaboration with the medical team

\section{(5) Assessing/ managing risks and symptoms:}

identifying and addressing the patient's priority risk factors, symptoms and health status as well as complete management of symptoms to prevent onset or risks

- In developing the care plan in close collaboration with the participant, his/her caregivers, and care team, the care plan includes the measures and activities planned and initiated by the hospital discharge planning team and integrates them with the ambulatory care measures. Additionally, the pathfinders make sure to respect and integrate the participant's individual preferences, values and goals.

- Assessing, identifying and managing risks and symptoms according to individual health status and situation is performed intensively, starting in the hospital and integrating the information of the hospital. The pathfinders_assessment is supported by a standardised questionnaire instrument based on the Neues Begutachtungs Assessment, an assessment to determine eligibility for benefits from the long-term care insurance in Germany, ${ }^{31}$ to identify individual care needs as well as to document and evaluate the needed or already initialised measures. The instrument assesses the participant's care situation, care supply and quality by examining the participant's living situation, mobility and falls, cognition, psychological situation, nutrition, self-support, medication, daily activities, housekeeping, vision and hearing capacities, continence, pain score, wound management, health and disease knowledgeability of participant and caregiver and caregiver burden. For each topic, the pathfinder evaluates whether or not there is a need for change, which measures would provide a remedy or whether or not already taken measures have helped to solve the problem or which amendments are needed. This instrument is applied at the first home visit and at visits T1, T2, T3 and T4.

- Additionally, the psychosomatic situation due to loneliness, grief for a deceased and depression, is assessed.

- Physical parameters recordings by the participant (eg, blood pressure, pain diary and weight log) are encouraged as a part of the intervention.

(6) Education/ promoting self-

management:

preparing older adults and family caregivers to identify and respond quickly to worsening symptoms, meeting their learning needs

\section{(7) Collaborating:}

promotes consensus on the plan of care between older adults and members of the care team
- Participants and their caregivers are provided with an emergency plan stating whom to contact in which case.

- Participants are encouraged and guided to recognise their own risks and symptoms, how to keep their health record updated and how to adequately contact physicians, health services, neighbourhood, and social networks, as a means of empowering self-reliant health management.

- Healthy behaviour regarding mobility, nutrition and prevention of the onset of symptoms or risks is regularly addressed by the pathfinders, and implementation of the participant's ideas for healthy behaviour is promoted.

- The pathfinders establish and facilitate efficient and trusting communication and consensus-building among the participant's care team partners: physicians within and outside of the hospital (family physicians of the participants), hospital care and discharge planning team, ambulant care services, medical store houses, occupational therapists, physiotherapists, nutritional therapists, charity networks, municipal organisations and also the participants and their caregivers themselves. 
Table 2 Continued

Intervention module/component as defined in TCM according to Hirschman et $\mathrm{a}^{22}$

(8) Promoting continuity: prevents breakdowns in care from hospital to home by having the same clinician involved across these sites. Promoting continuity could help to prevent breakdowns in care across settings

(9) Fostering coordination: promotes and encourages communication and connections between the healthcare team and community-based practitioners
- The same pathfinder gets to know the participant and his/her situation in the hospital, establishes and advances the care plan within the care team in the hospital, at transition, and in the homesetting. The intense follow-up in the first month (see figure 1) and the follow-up of 6-12 months promote continuity.

- In the case of readmission of the participant, the pathfinder supports continuity of care and information transfer from the ambulant care setting back into the hospital BBR again.

- The pathfinder coordinates and fosters information exchange in the care team in the inpatient and in the home-setting, as well as across settings and regarding municipal or health insurance health course offerings.

- Especially, the municipal and charity offerings of the city of Regensburg are manifold, but mostly not networked. The pathfinders help to connect the participants with suitable public offerings.

APRN, advanced practice registered nurses; BBR, Barmherzige Brüder Regensburg; eCRF, electronic case report form; TCM, transitional care model; TIGER, Transsectoral Intervention Programme for Improvement of Geriatric Care in Regensburg.

criterion. The IG participants are visited by the pathfinders and contacted by telephone. The individual care plan is regularly evaluated in the home visits (at least two times a month in the first month after discharge, at least once in a month in the second and third month after discharge) and telephone calls (at least two times in the first month after discharge and at least once in a month in all the following months until the end of study visit).

The pathfinders' work is supported by a standardised questionnaire instrument based on the 'Neues Begutachtungs Assessment', an assessment to determine eligibility for benefits from the long-term care insurance in Germany, ${ }^{31}$ to identify individual care needs out of a broad range of possible care needs as well as to document and evaluate the needed or already initialised measures (for details on the spectrum of specified care needs see table 2).

In the IG, the standardised assessments (see section 'Assessments of both groups') take place at the beginning (T0), after 1 month (T1), 3 months (T2), 6 months (T3) and at the end of study (T4).

\section{Control group}

The control group (CG) receives usual hospital discharge planning by hospital staff not related to the TIGER study and usual ambulatory care after discharge. Usual discharge planning involves the first initiation of procurement of therapeutic adjuvants or appliances after hospital discharge, taking the hospital information, and if possible, a conversation with the patient and a caregiver into account. Medication for the first few days after discharge is supplied. No verifications of the arrangements at home are possible; the family physician of the patient is not contacted. No measures are initiated associated with the TIGER study.

The CG is assessed (see section 'Assessments in both groups') by the TIGER study nurses at the beginning (T0), after 3 months (T2) and at the end of the study (T4). It fills out the standardised questionnaires for participants also at T1 (after 1 month) and T3 (after 6 months) (see table 1).

\section{Assessments in both groups}

All study participants receive regular standardised assessments at visits T0-T4 using validated instruments (see table 1) to assess health and care degree, functionality and mobility, nutritional status, geriatric and cognitive situation, and domestic care situation. Since a comprehensive geriatric assessment is not mandatory in all wards at hospital admission of an older patient, it is administered in the TIGER study directly at recruitment and up to four times after discharge (depending on IG/CG and duration of participation: 6-12 months).

Additionally, health-related quality of life, personal psychosocial resources regarding health, wound-related quality of life, stress perception, as well as the burden of informal caregivers, are assessed by questionnaires to be filled out by the study participants autonomously. Table 1 gives an overview of when and which assessments are performed by the pathfinders and study nurses in the IG and the CG, respectively.

If the family physician of an IG participant agrees to participate in the study, he or she performs the geriatric assessments for the study (see table 1) at visits T1-T4 (see figure 1). Otherwise, the geriatric assessments at visits T1T4 are performed by the study physician or pathfinders for the IG, and by the study physician or study nurse for the CG.

\section{Outcomes}

The primary outcome is the readmission rate, since the application of TCM in the USA has shown to reduce the readmission rate as a major negative outcome for geriatric patients leaving the hospital. ${ }^{25}{ }^{27}$ Readmission rate is defined as the proportion of patients who have at least one unplanned readmission into any hospital (not rehabilitation clinic) within a follow-up of up to 12 months after hospital discharge, using anonymised data of the health insurance fund AOK Bavaria.

Secondary outcomes include care situation, care supply, and quality at home, functionality, and mobility, nutritional status, geriatric assessment-outcomes (depression and cognitive status, and ADL), questionnaires for 
participants outcomes (wound condition, health-related quality of life, psychosocial resources of participants regarding health and burden of informal caregivers), frequency of transfers into nursing homes. A detailed description of all assessment instruments and time points of data collection, including quotes, is depicted in table 1.

Another important secondary outcome is the efficiency of the pathfinder intervention. In a cost-cost analysis, costs of both groups (eg, intervention costs and healthcare costs) will be compared, to assess if the intervention leads to monetary savings. In an additional cost-utility analysis, costs of the intervention will be compared in both groups to non-monetary benefits (eg, higher quality of life).

\section{Sample size}

Based on international studies, ${ }^{21} 2527$ it can be assumed that the implementation of a transitional care concept can reduce the readmission rate by $40 \%$. An analysis of AOK Bayern data of 2018 points to a readmission rate of approximately $41.9 \%$ for people above 75 years of age in Germany. Thus, we expect the general readmission rate of $41.9 \%$ to drop by $40 \%$ to a readmission rate of about $25.1 \%$ by the intervention. We aim at determining this effect with a level of significance of $5 \%$ and a power of at least $80 \%$ with the intervention and control group of equal size. Therefore, 280 intention to treat participants in total are calculated to be sufficient to reach a statistically significant validation and statement based on the assumptions stated above, and taking a loss-to-follow-up rate of $15 \%-20 \%$ into account.

\section{Data collection and monitoring methods}

The recruitment period started with the first-patient-in on 25 April 2018 and ended on 31 December 2019. The pathfinders, study nurses and partly the study physician and/or participants' family physicians collect the assessment and questionnaire data on paper forms at visits T0, T1, T2, T3 and T4, respectively, for each participant (see table 1) and before data entry into the eCRF. Any change in care setting, form or participation is documented in a standardised format (eg, necessary readmission with entry date and discharge date, commission to nursing home, participant's wish to discontinue and death) for subsequent evaluation.

For monitoring data quality and completeness, double data entry is performed for the assessments. Data from the paper forms are entered into the eCRF by two different eCRF-trained research associates in two input forms. In case of differences between the two versions of data input, the original paper file is checked, and the entry corrected accordingly.

Once a month, the trial site, pathfinders and study nurses are monitored by the project coordinator. Trial conduct and recruitment are discussed, possible obstacles identified and other consortium partners are invited to the meetings if indicated. Problems in performance, quality, medical or other issues are analysed, solutions initiated and implemented. Consequent protocol of operations amendments are implemented, and new protocol versions distributed within the consortium. The ethics committee is informed on protocol amendments regarding ethical issues. Amendments are introduced into the clinical trials.gov file.

\section{Statistical methods}

The primary outcome hospital readmission rate will be evaluated by Fisher's exact test. The main analysis will be performed for the first 3 months follow-up of patients after discharge. In addition, a subanalysis will be performed with data after 6 months, 9 months and 12 months follow-up. Possible interactions with housing situation, availability of caring relatives and risk factors like care dependency or limitations in cognition will be analysed by multiple regression for a better understanding of the intervention's impact on the hospital readmission rate.

Secondary outcomes (eg, quality of life and mobility) will be analysed by t-test, Fisher's test, Mann-Whitney test or $\chi^{2}$ test, depending on distribution and number of cases. Subgroup analysis will be performed for primary and secondary outcomes, for example, for participants with or without caregivers, for participants with risk of malnutrition and for participants with different classifications from long-term care insurance.

Repeated measurements will be analysed by Analysis of Variance (ANOVA) or linear mixed model.

To deal with missing data, for the primary outcome, complete case analysis will be applied. In case of the secondary outcomes, either complete case analysis or, if appropriate and applicable, multiple imputation will be considered.

The analysis of possible financial benefits of implementing a pathfinder will be carried out by t-test. The main analyses will be performed using SPSS (IBM SPSS Statistics 26) and R.

\section{Patient and public involvement}

One of the TCM's activities and a part of this study's design is the engagement of patients and their caregivers in their own care planning, so every participant is actively involved in the construction of his/her own care planning and in the conduct of the study. Progressively during the course of the intervention, self-management of the participants is promoted.

\section{Confidentiality}

The signed ICF of the participants are stored in BBR separate from the assessed data of the participants, anonymised with the participants' eCRF IDs. The list that contains both participants' names and their eCRF IDs is stored on a stand-alone PC without internet connection, being deleted after the study. Original paper files are archived according to data protection regulations of Bavaria, Germany. 


\section{ETHICS AND DISSEMINATION}

\section{Research ethics approval}

The ethical committee of Friedrich-Alexander Universität Erlangen-Nürnberg approved the study on 5 March 2018 (\# 60-18 B) prior to first participant inclusion. The study will be conducted in accordance with the Declaration of Helsinki.

\section{Harms}

We expect no harm due to the study since all interventions are performed by experienced professionals, and medical as well as nursing care of participants remain proficient.

\section{Access to data}

The final anonymised trial data set will be available to the TIGER consortium. After completion of evaluation and dissemination by the TIGER consortium, the anonymised data set will be available to interested researchers on demand.

\section{Ancillary and post-trial care}

CG participants are provided at the end of study visit with a short explanation and feedback letter concerning the development of their health scores throughout the study regarding MMSE, Geriatric Depression Score, ${ }^{32}$ Mini Nutritional Assessment, ${ }^{33} 34$ as well as handgrip strength (see table 1) over time. Each IG participant receives a list of the contacted ambulatory services helpful during the intervention at the end of study visit.

\section{Dissemination policy}

The study was registered at clinical trials.gov. According to outcomes, the results will be published in peer-reviewed scientific journals and disseminated in the geriatric and nursing care specialised healthcare communities, to promote effective intervention activities for the process of introduction into regular healthcare.

\section{DISCUSSION}

Local adaptations of the TCM were described as being necessary by the architects of the model themselves. ${ }^{35}$ The application of the TCM modules within the TIGER trial in Germany also needs adaptations, mostly contextual in nature (see table 2) or due to the German healthcare settings. For the module 'Collaborating', the family physicians for the intervention participants can actively take part in the study performing the geriatric assessments and having regular information exchange with the pathfinder on behalf of the joint participant. This integration of family physicians directly in the study design stresses their essential role not only in post-discharge management of the patient, but underlining the transsectoral importance of a collaborative team.

We experienced a considerable lag behind the planned recruitment. This encountered recruitment difficulty is also reported in other clinical trials that engage persons over 65 years of age. Discussed reasons for higher challenges of recruitment of this vulnerable patient group are their feeling unwell at recruitment, too long and detailed participant information sheets and not wishing to take advice from additional healthcare persons or to have visits at home. ${ }^{28}$

To address recruitment difficulties, we introduced from the beginning a face-to-face and stepwise approach of information provision of study content to participants, as recommended. ${ }^{28}$ Adaptations and optimisations of the recruitment process were implemented to maximise recruitment success. Thus, we expanded the included wards from originally three wards to all wards of the hospital, that could harbour eligible TIGER participants. In case the questionnaires for participants would be a too high burden and thus an impediment to participate, we allowed participants to take part and only join the assessments and intervention. Also, the recruitment schedule and follow-up visit plan were adapted. In our original study design, a follow-up of up to 1 year after discharge was targeted ${ }^{27}$ for a comprehensive evaluation, offering a long time benefit to the participants. Since a minimum follow-up of 6 months has been discussed as also being effective in reducing readmission rates significantly in patients over 65 years of age, ${ }^{21} 3637$ while lowering healthcare costs, the adapted study design prolongs the recruitment phase (from 12 months to 20 months) allowing for a follow-up of at least 6 months for those participants recruited after June 2019 (see figure 1).

According to Greysen and colleagues, ${ }^{38}$ participants who are more fragile (poorer physical function, older age, suffering from multimorbidity, impairment in ADL, etc) are the ones who tend to present higher readmission rates. Since there is still a gap of knowledge on the efficiency of TCM concepts for geriatric patients significantly older than 65 years of age, it will be interesting to see which participants in the TIGER study profit most from the intervention. The detailed analysis of assessments of mobility and functionality, nutrition, geriatric issues and wound situation of the TIGER study will shed light on the most needed areas of intervention for this vulnerable patient group, even if the readmission rates of this patient group $\geq 75$ years of age with chronic disease might not be reduced as much by the intervention as anticipated.

In general, this study shows a wide scope of combined qualitative and quantitative analyses of the care situation of geriatric patients, of influencing factors and of the impact of pathfinders activities on readmission rate. The study will provide important additional data on the TCM component implementation over different time periods ranging from 4 weeks to 12 months. On a national level, it will add knowledge concerning if and how a transitional care concept or parts of it can also be applied in Germany with its fragmented established structures in order to define necessary steps to improve continuous transitional care for the geriatric patient group analysed in this study.

For patients with cognitive deficits, further transitional care intervention studies need to be conducted. 
In case of a positive evaluation regarding its scientific and health-economic outcomes, a prospective goal is to define clear implementation possibilities of pathfinder activities for the analysed patient group in the German healthcare system.

\section{Author affiliations}

${ }^{1}$ Institute for Biomedicine of Aging, Friedrich-Alexander-Universität Erlangen-

Nürnberg, Nuremberg, Germany

${ }^{2}$ St John of God Hospital, Regensburg, Germany

${ }^{3}$ AOK Bavaria, Munich, Germany

${ }^{4}$ Federal Association of Geriatrics, Berlin, Germany

${ }^{5}$ Regensburger Ärztenetz, Regensburg, Germany

${ }^{6}$ Chair of Health Management, Friedrich-Alexander-Universität Erlangen-Nürnberg, Nuremberg, Germany

${ }^{7}$ Institute for Nursing Science at the University of Bielefeld, Bielefeld, Germany ${ }^{8}$ Department of Social Medicine and Prevention, Faculty of Medicine, Institute for Community Medicine, University of Greifswald, Greifswald, Germany

${ }^{9}$ Kantonsspital Winterthur, Winterthur, Switzerland

Acknowledgements Principal investigator and project coordinator is the Institute for Biomedicine of Ageing. The Federal Association of Geriatrics reviews current projects in Germany, including geriatric patients who are discharged from hospitals for the project, and will disseminate results in interacting with healthcare stakeholders. The intervention takes place at BBR in collaboration with the Regensburger Aerztenetz. The health insurance fund AOK Bavaria will provide anonymous routine data for evaluation. Psychogerontological advice is provided by the chair for social medicine and prevention at University Medicine Greifswald. The scientific and health-economic evaluation will be performed by the Institute for Nursing Science at the University of Bielefeld, and the chair of health management at the University Erlangen-Nürnberg, respectively.We thank the TIGER (Transsectoral Intervention Programme for Improvement of Geriatric Care in Regensburg) staff Sarah Blaimer, Teresa Freund, TG, Claudia Gradl, Sylvie Lamotte, Renate Roetzer, Christina Schrader and Manfred Seitz, and the study physician Stephan Theobaldy for their relentless recruiting efforts and ongoing finding of individual best care paths.

Contributors MR: project coordination, study supervision, improvements of study design and writing the original manuscript. JW: partaking in original study design and partaking in original manuscript writing. SB: drafting manuscript figures and revising the manuscript. TG: improvements in study design and revising the manuscript. MH, DvdH, AK, TK, OS, DV, KW and SW: partaking in original study design and revising the manuscript. EF: conceptualisation of study and study design, and revising the manuscript. CS: conceptualisation of study and study design, revising the manuscript and principal investigator.

Funding The project TIGER (Transsectoral Intervention Programme for Improvement of Geriatric Care in Regensburg) is funded by the Innovation Fund at the Federal Joint Committee of Germany (https://www.g-ba.de/english) with the funding number 01NVF17005.

Competing interests None declared.

Patient consent for publication Not required.

Provenance and peer review Not commissioned; externally peer reviewed.

Supplemental material This content has been supplied by the author(s). It has not been vetted by BMJ Publishing Group Limited (BMJ) and may not have been peer-reviewed. Any opinions or recommendations discussed are solely those of the author(s) and are not endorsed by BMJ. BMJ disclaims all liability and responsibility arising from any reliance placed on the content. Where the content includes any translated material, BMJ does not warrant the accuracy and reliability of the translations (including but not limited to local regulations, clinical guidelines, terminology, drug names and drug dosages), and is not responsible for any error and/or omissions arising from translation and adaptation or otherwise.

Open access This is an open access article distributed in accordance with the Creative Commons Attribution Non Commercial (CC BY-NC 4.0) license, which permits others to distribute, remix, adapt, build upon this work non-commercially, and license their derivative works on different terms, provided the original work is properly cited, appropriate credit is given, any changes made indicated, and the use is non-commercial. See: http://creativecommons.org/licenses/by-nc/4.0/.
ORCID iDs

Martina Rimmele http://orcid.org/0000-0001-6759-4681

Ellen Freiberger http://orcid.org/0000-0002-6506-6276

\section{REFERENCES}

1 Eurostat. Ageing Europe_Looking at the lives of older people in the EU, 2019. Available: https://ec.europa.eu/eurostat/statisticsexplained

2 Yarnall AJ, Sayer AA, Clegg A, et al. New horizons in multimorbidity in older adults. Age Ageing 2017;46:882-8.

3 Sasseville M, Smith SM, Freyne L, et al. Predicting poorer health outcomes in older community-dwelling patients with multimorbidity: prospective cohort study assessing the accuracy of different multimorbidity definitions. BMJ Open 2019;9:e023919.

4 Gruneir A, Bronskill SE, Maxwell CJ, et al. The association between multimorbidity and hospitalization is modified by individual demographics and physician continuity of care: a retrospective cohort study. BMC Health Serv Res 2016;16:154.

5 Severinsen KD, Tufton A, Hannan E, et al. Evaluating outcomes from an integrated health service for older patients. Ochsner $J$ 2015;15:423-8.

6 Admi H, Shadmi E, Baruch $\mathrm{H}$, et al. From research to reality: minimizing the effects of hospitalization on older adults. Rambam Maimonides Med J 2015;6:e0017.

7 Groene RO, Orrego C, Suñol R, et al. "It's like two worlds apart": an analysis of vulnerable patient handover practices at discharge from hospital. BMJ Qual Saf 2012;21 Suppl 1:i67-75.

8 Jencks SF, Williams MV, Coleman EA. Rehospitalizations among patients in the Medicare fee-for-service program. N Engl J Med 2009;360:1418-28.

9 Field TS, Ogarek J, Garber L, et al. Association of early postdischarge follow-up by a primary care physician and 30day rehospitalization among older adults. J Gen Intern Med 2015;30:565-71.

10 Roeper B, Beck E, Castillo D, et al. Innovative care model to improve clinical quality and safety of transitional care: early outcomes. Manag Care 2017;26:35-8.

11 Gesundheitswesen DDVfSAi. DVSG Positionspapier. Entlassungsmanagement durch Soziale Arbeit in Krankenhäusern und Rehabilitationskliniken Berlin: Deutsche Vereinigung für Soziale Arbeit Im Gesundheitswesen e.V, 2013.

12 Lehnert O, Müller M. Neue Wege Im Entlassmanagement. Kosten reduzieren, Erlöse sichern, Versorgung gestalten. professional process. 2, 2011

13 Deimel D, Kuß A, Ossege M. Positionspapier 'Entlassmanagement im Krankenhaus'. Berlin: Bundesverband Managed Care e V, 2012.

14 Blum K, Reus U. Entlassungsmanagement Im Krankenhaus - eine Repräsentativbefragung deutscher Krankenhäuser. Das Krankenhaus 2014;7:8.

15 Freund T, Campbell SM, Geissler S, et al. Strategies for reducing potentially avoidable hospitalizations for ambulatory care-sensitive conditions. Ann Fam Med 2013;11:363-70.

16 Hesselink G, Flink M, Olsson M, et al. Are patients discharged with care? A qualitative study of perceptions and experiences of patients, family members and care providers. BMJ Qual Saf 2012;21 Suppl 1:i39-49.

17 Bundesverband Geriatrie eV. WeiBbuch Geriatrie - Die Versorgung geriatrischer Patienten: Strukturen und Bedarf- Status Quo und Weiterentwicklung. In: Eine analyse durch die GEBERA Gesellschaft für betriebswirtschaftliche Beratung $\mathrm{mbH}$. e.V. eBG. Stuttgart: Kohlhammer-Verlag, 2010.

18 Benbassat J, Taragin M. Hospital readmissions as a measure of quality of health care: advantages and limitations. Arch Intern Med 2000;160:1074-81.

19 Allen J, Ottmann G, Roberts G. Multi-professional communication for older people in transitional care: a review of the literature. Int $\mathrm{J}$ Older People Nurs 2013;8:253-69.

20 Leyenaar JK, O'Brien ER, Leslie LK, et al. Families' priorities regarding Hospital-to-Home transitions for children with medical complexity. Pediatrics 2017;139:peds.2016-1581. doi:10.1542/ peds.2016-1581

21 Naylor MD, Brooten D, Campbell R, et al. Comprehensive discharge planning and home follow-up of hospitalized elders: a randomized clinical trial. JAMA 1999;281:613-20.

22 Hirschman KB, Shaid E, McCauley K, et al. Continuity of care: the transitional care model. Online J Issues Nurs 2015;20:1.

23 Coleman EA, Parry C, Chalmers S, et al. The care transitions intervention: results of a randomized controlled trial. Arch Intern Med 2006;166:1822-8. 
24 Verhaegh KJ, MacNeil-Vroomen JL, Eslami S, et al. Transitional care interventions prevent Hospital readmissions for adults with chronic illnesses. Health Aff 2014;33:1531-9.

25 Naylor M, Brooten D, Jones R, et al. Comprehensive discharge planning for the hospitalized elderly. A randomized clinical trial. Ann Intern Med 1994;120:999-1006.

26 Naylor MD, Aiken LH, Kurtzman ET, et al. The care span: the importance of transitional care in achieving health reform. Health Aff 2011:30:746-54.

27 Naylor MD, Brooten DA, Campbell RL, et al. Transitional care of older adults hospitalized with heart failure: a randomized, controlled trial. $J$ Am Geriatr Soc 2004;52:675-84.

28 Piantadosi C, Chapman IM, Naganathan V, et al. Recruiting older people at nutritional risk for clinical trials: what have we learned? BMC Res Notes 2015;8:151.

29 Folstein MF, Folstein SE, McHugh PR. "Mini-mental state". A practical method for grading the cognitive state of patients for the clinician. J Psychiatr Res 1975;12:189-98.

30 Hardy SE, Kang Y, Studenski SA, et al. Ability to walk 1/4 mile predicts subsequent disability, mortality, and health care costs. $J$ Gen Intern Med 2011;26:130-5.

31 Büscher A, Wingenfeld K, Schaeffer D. Determining eligibility for long-term care-lessons from Germany. Int J Integr Care 2011;11:e019.

32 Yesavage JA. Geriatric depression scale. Psychopharmacol Bull 1988;24:709-11.

33 Guigoz Y, Vellas B, Garry PJ. Assessing the nutritional status of the elderly: the mini nutritional assessment as part of the geriatric evaluation. Nutr Rev 1996;54:S59-65.

34 Kaiser MJ, Bauer JM, Ramsch C, et al. Validation of the mini nutritional assessment short-form (MNA-SF): a practical tool for identification of nutritional status. J Nutr Health Aging 2009;13:782-8.

35 Naylor MD, Hirschman KB, Toles MP, et al. Adaptations of the evidence-based transitional care model in the U.S. Soc Sci Med 2018;213:28-36.

36 Rich MW, Beckham V, Wittenberg C, et al. A multidisciplinary intervention to prevent the readmission of elderly patients with congestive heart failure. N Engl J Med 1995;333:1190-5.

37 López Cabezas C, Falces Salvador C, Cubí Quadrada D, et al. Randomized clinical trial of a postdischarge pharmaceutical care program vs regular follow-up in patients with heart failure. Farm Hosp 2006;30:328-42.

38 Greysen SR, Stijacic Cenzer I, Auerbach AD, et al. Functional impairment and hospital readmission in Medicare seniors. JAMA Intern Med 2015;175:559-65.

39 Guralnik JM, Simonsick EM, Ferrucci L, et al. A short physical performance battery assessing lower extremity function: association with self-reported disability and prediction of mortality and nursing home admission. J Gerontol 1994;49:M85-94.

40 Army U. Army individual test battery. manual of directions and scoring, 1944.
41 Mahoney FI, Barthel DW. Functional evaluation: the Barthel index: a simple index of independence useful in scoring improvement in the rehabilitation of the chronically ill. Md State Med J 1965.

42 Podsiadlo D, Richardson S. The timed "Up \& Go": a test of basic functional mobility for frail elderly persons. J Am Geriatr Soc 1991;39:142-8

43 Lawton MP, Brody EM. Assessment of older people: selfmaintaining and instrumental activities of daily living. Gerontologist 1969;9:179-86.

44 Morfeld M, Kirchberger I, Bullinger M. SF-36 Fragebogen zum Gesundheitszustand: Deutsche version des short Form-36 health survey: Hogrefe Verlag, 2011.

45 Friedman SM, Munoz B, West SK, et al. Falls and fear of falling: which comes first? A longitudinal prediction model suggests strategies for primary and secondary prevention. J Am Geriatr Soc 2002;50:1329-35.

46 Schwenk M, Lauenroth A, Stock C, et al. Definitions and methods of measuring and reporting on injurious falls in randomised controlled fall prevention trials: a systematic review. BMC Med Res Methodol 2012;12:50.

47 Dias N, Kempen GIJM, Todd CJ, et al. The German version of the Falls Efficacy Scale-International Version (FES-I)]. Z Gerontol Geriatr 2006;39:297-300.

48 Freund AM, Baltes PB. Life-management strategies of selection, optimization, and compensation: measurement by self-report and construct validity. J Pers Soc Psychol 2002;82:642-62.

49 Dittmann-Kohli F, Kohli M, Künemund H. Lebenszusammenhänge, Selbst-und Lebenskonzeptionen - Erhebungsdesign und Instrumente des Alters-Survey. Forschungsgruppe Altern und Lebenslauf (FALL) [Internet]. Forschungsbericht 61, 1997. Available: http://fall-berlin.de/ index.html?/fdp7.htm

50 Jerusalem M, Schwarzer R. Allgemeine Selbstwirksamkeit. Skalen Zur Erfassung von Lehrer-und Schülermerkmalen. 13, 1999. http:// www.psyc.de/skalendoku.pdf

51 Wilson M-MG, Thomas DR, Rubenstein LZ, et al. Appetite assessment: simple appetite questionnaire predicts weight loss in community-dwelling adults and nursing home residents. Am J Clin Nutr 2005;82:1074-81.

52 Vailas LI, Nitzke SA, Becker M, et al. Risk indicators for malnutrition are associated inversely with quality of life for participants in meal programs for older adults. J Am Diet Assoc 1998;98:548-53.

53 Augustin M, Baade K, Herberger K, et al. Use of the WoundQoL instrument in routine practice: feasibility, validity and development of an implementation tool. Wound Medicine 2014;5:4-8.

54 Fliege $\mathrm{H}$, Rose M, Arck $\mathrm{P}$, et al. The perceived stress questionnaire (PSQ) reconsidered: validation and reference values from different clinical and healthy adult samples. Psychosom Med 2005;67:78-88.

55 Zarit SH, Reever KE, Bach-Peterson J. Relatives of the impaired elderly: correlates of feelings of burden. Gerontologist 1980;20:649-55

56 Braun M, Scholz U, Hornung R, et al. [Caregiver burden with dementia patients. A validation study of the German language version of the Zarit Burden Interview]. Z Gerontol Geriatr 2010;43:111-9. 